\title{
MiR-27a Promotes Hemin-Induced Erythroid Differentiation of K562 Cells by Targeting CDC25B
}

\author{
Dongsheng Wang ${ }^{a}$ Si Sib ${ }^{b}$ Qiang Wang ${ }^{a}$ Guangcheng Luo ${ }^{a} \quad$ Qin Du ${ }^{a} \quad$ Qi Liang ${ }^{a}$ \\ Xiaolan Guo a,d Guoyuan Zhang ${ }^{\mathrm{a}}$ Jiafu Fenge Zhengwei Leng ${ }^{\mathrm{c}}$ \\ aDepartment of Laboratory Medicine, Affiliated Hospital of North Sichuan Medical College, Nanchong, \\ bDepartment of Blood Transfusion, Nanchong Central Hospital, Nanchong, 'Department of General \\ Surgery, Affiliated Hospital of North Sichuan Med. I College, Nanchong, Sichuan, 'Translational \\ Medicine Center, North Sichuan Medical College, Nanchong, eDepartment of Laboratory Medicine, \\ Mianyang Central Hospital, Mianyang, PR China
}

\section{Key Words}

Microrna • Erythropoiesis • MiR-27a • CDC25B

\begin{abstract}
Background/Aims: MicroRNAs (miRNAs) play a crucial role in erythropoiesis. MiR23a 27a 24-2 clusters have been proven to take part in erythropoiesis via some proteins. CDC25B (cell division control Cdc2 phosphostase B) is also the target of mir-27a; whether it regulates erythropoiesis and its mechanism are unknown. Methods: To evaluate the potential role of miR-27a during erythroid differentiation, we performed miR-27a gain- and loss-offunction experiments on hemin-induced $\mathrm{K} 562$ cells. We detected miR-27a expression after hemin stimulation at different time points. At the same time, the $\gamma$-globin gene also was measured via real-time PCR. According to the results of the chips, we screened the target protein of miR-27a through a dual-luciferase reporter assay and identified it via Western blot analyses. To evaluate the function of CDC25B, benzidine staining and flow cytometry were employed to detect the cell differentiation and cell cycle. Results: We found that miR27a promotes hemin-induced erythroid differentiation of human K562 cells by targeting cell division cycle 25 B (CDC25B). Overexpression of miR-27a promotes the differentiation of hemininduced K562 cells, as demonstrated by $\gamma$-globin overexpression. The inhibition of miR-27a expression suppresses erythroid differentiation, thus leading to a reduction in the $\gamma$-globin gene. CDC25B was identified as a new target of miR-27a during erythroid differentiation. Overexpression of miR-27a led to decreased CDC25B expression after hemin treatment, and CDC25B was up-regulated when miR-27a expression was inhibited. Moreover, the inhibition of CDC25B affected erythroid differentiation, as assessed by $Y$-globin expression. Conclusion: This study is the first report of the interaction between miR-27a and CDC25B, and it improves the understanding of miRNA functions during erythroid differentiation.

W. Dongsheng, S. Si and W. Qiang contributed equally to this work.

Feng jiafu

and Leng Zhengwei

Dpt. of Laboratory Medicine, Mianyang Central Hospital, Mianyang, 621099 (China)

Dpt. of General Surgery, Affiliated Hospital of North Sichuan Med.I College, Nanchong, Sichuan (China); E-Mail jiafufeng@aliyun.com, lengzhengwei@163.com
\end{abstract}

\section{KARGER}




\section{Cellular Physiology Cell Physiol Biochem 2018;46:365-374 and Biochemistry Published online: March 27, $2018 \quad$\begin{tabular}{l|l} 
DOI: 10.1159/000488436 2018 The Author(s). Published by S. Karger AG, Basel \\
www.karger.com/cpb
\end{tabular}}

Wang et al.: MiR-27a Promotes Erythroid Differentiation by Targeting CDC25B

\section{Introduction}

MicroRNAs (miRNAs) are a class of small non-coding linear regulatory RNAs that posttranscriptionally regulate gene expression, including both mRNA degradation and protein translation inhibition. Indeed, miRNAs play important roles in the regulation of a variety of biological processes, such as embryonic development, cell proliferation, and differentiation [1-3]. Recently, the critical regulatory role of miRNAs in hematopoiesis and their important role in the differentiation of specific lineages have been demonstrated $[4,5]$.

Erythropoiesis is a hematopoietic process that is tightly regulated by cell lineage specification, proliferation, and differentiation $[4,6]$. To date, a number of miRNAs, such as miR-15a, miR-16, miR-23a, miR-126, miR-144, miR-210, miR-221, miR-223, miR-376a and miR-451, have been shown to play critical roles in controlling erythropoiesis, including regulating the proliferation and maturation of early erythroid cells and the expression of fetal $\gamma$-globin genes during erythroid differentiation [7, 8]. Previously, Zhu et al. reported that miR-23a is a positive erythroid regulator that is activated by GATA- 1 during erythroid differentiation [9]. Moreover, Ma's study has demonstrated that miR-23a promotes the expression and transcription of the $\beta$-like globin gene by targeting the transcription factors KLF3 and SP1 during the erythroid differentiation of K562 cells [10]. Recently, Zhang et al. found that the miR-23a-27a-24 cluster is expressed in the APL and AML cell lines and nucleated peripheral blood cells from leukemia patients and is up-regulated by NF- $\mathrm{B}$ p65 in erythroleukemia K562 cells [11]. Furthermore, Su and colleagues have reported that miR23a, miR-27a and miR-24 synergistically regulate the JAK1/Stat3 cascade and serve as novel therapeutic targets in human acute erythroid leukemia [12]. Accumulating evidence suggests that this cluster of genes is derived from a common ancestor and often exhibits similar features in cellular processes. Because miR-23a plays a critical role in erythropoiesis, and miR-23a, miR-27a and miR-24 are derived from a common gene cluster, further investigation is required to determine the regulatory and functional significance of miR-27a in erythroid differentiation.

The cell division cycle 25 (CDC25) phosphatases include three isoforms, i.e., CDC25A, CDC25B and CDC25C, which are highly conserved dual-specificity phosphatases that dephosphorylate and activate cyclin-dependent kinase (CDK) complexes [13]. CDC25B, which mainly activates CDK1-cyclin B at the G2-M transition phase, has also been demonstrated to be recruited to the mother centrosome and to be involved in the centrosome duplication cycle and microtubule nucleation [14]. The overexpression of CDC25B correlates with malignant disease and poor prognosis in certain malignancies and leads to genetic instability in mice $[13,15,16]$. In addition, CDC25B is a key regulator of the cell cycle in red blood cell (RBC) precursors and is downregulated in the bone marrow, owing to the inhibition of erythropoiesis after myelosuppression treatment [17]. CDC25B is bioinformatically predicted to be a direct target of miR-27a, and it contains a complete complementary motif with miR-27a at the 3' UTR of its mRNA.

Based on the abovementioned literature, we hypothesized that miR-27a and CDC25B may be involved in erythropoiesis. However, whether miR-27a and CDC25B are indispensable in erythropoiesis and the mechanism by which they function during erythroid differentiation regulation remain unknown. In this study, we focused on the regulation of the hemininduced erythroid differentiation of K562 cells using miR-27a gain- and loss-of-function experiments to elucidate the related mechanisms. We confirmed that miR-27a plays a novel role in modulating erythroid differentiation via CDC25B, which was identified and validated as a target of miR-27a. This study is the first report highlighting the relationship between miR$27 \mathrm{a}$ and CDC25B in erythropoiesis, and it provides new insight into erythroid differentiation.

\section{Materials and Methods}

Culture conditions

Human leukemia K562 cells (ATCC CCL-243) were obtained from the American Type Culture Collection (ATCC, Maryland Rockefeller, MD, USA). The K562 cells were cultured in RPMI 1640 media (Sigma, St 


\section{Cellular Physiology Cell Physiol Biochem 2018;46:365-374 and Biochemistry Published online: March 27, $2018 \quad \begin{aligned} & \text { DOI: 10.1159/000488436 } 2018 \text { The Author(s). Published by S. Karger AG, Basel } \\ & \text { www.karger.com/cpb }\end{aligned}$ \\ Wang et al.: MiR-27a Promotes Erythroid Differentiation by Targeting CDC25B}

Louis, MO, USA) supplemented with 10\% FBS (Sigma, St Louis, MO, USA) at $37{ }^{\circ} \mathrm{C}$ in $5 \% \mathrm{CO}_{2}$. Hemin (30 $\mu \mathrm{M}$, Sigma) was used to induce the erythroid differentiation of the K562 cells. HEK293 cells (ATCC CRL-1573) were grown in DMEM (Sigma) with 10\% FBS at $37^{\circ} \mathrm{C}$ in $5 \% \mathrm{CO}_{2}$.

Oligonucleotides, plasmid construct and cell transduction

The miR-27a mimic, mimic control, miR-27a
Table 1. Human specific PCR primers

\begin{tabular}{lcc}
\hline Gene & Forward primer $\left(5^{\prime}->3^{\prime}\right)$ & Reverse primer $\left(5^{\prime}>3^{\prime}\right)$ \\
\hline miR-27a & TGCGCTTCACAGTGGCTAAG & CAGTGCAGGGTCCAGGT \\
U6 & CTCGCTTCGGCAGCACATATACT & ACGCTTCACGAATTTGCGTGTC \\
ã-globin & GCAGCTTGTCACAGTGCAGTTC & TGGCAAGAAGGTGCTGACTTC \\
CDC25B & GAGAAACCCTGGGAAGGCTC & TGTGCCTTGACTTTGGGGTT \\
GAPDH & TCAACGACCACTTTGTCAAGCTCA & GCTGGTGGTCCAGGGGTCTTACT \\
miR-27a & GTCGTATCCAGTGCAGGGTCCGAGGT \\
RTprimer & ATTCGCACTGGATACGACGCGGAA \\
U6 RTprimer & AAAATATGGAACGCTTCACGAATTG \\
\hline
\end{tabular}

inhibitor and inhibitor control were obtained from Dharmacon (Dharmacon, Lafayette, CO, USA). The K562 cells were transiently transduced with these oligonucleotides at a final concentration of $60 \mathrm{nM}$. and $1 \mu \mathrm{g}$ PGL3-CDC25B plasmid using Lipofectamine-2000 (Invitrogen, Paisley, UK) according to the manufacturer's instructions. The cells were harvested at $0,3,6,12,24$ and 48 hours post-transfection and subjected to various analyses. The PGL3-CDC25B plasmid, miR-27a mimic, mimic control, miR-27a inhibitor and inhibitor control were transduced into HEK293 cells under the same conditions. The 3' UTRs of CDC25B were first amplified using PCR primers (Table 1) and then cloned into the PGL3-basic reporter plasmid (Promega, Madison, WI, USA).

\section{RNA extraction and quantitative real-time RT-PCR}

Total RNA was isolated with TRIzol reagent (Invitrogen). cDNA was synthesized with a Revert Aid First Strand cDNA Synthesis Kit (Thermo Scientific, Waltham, MA, USA). The RT reaction for miR-27a quantification was performed with stem-loop RT primers according to the manufacturer's instructions (RiboBio, Guangzhou, China). U6 was used as an internal control. The relative quantification was calculated using the $2-{ }^{\Delta \Delta} \mathrm{Ct}$ formula. $\gamma$-globin and CDC25B were quantified with real-time PCR. Quantitative PCR was performed using SYBR Green qPCR Master Mixes (Takara, Dalian, China). GAPDH (primers from RiboBio, Guangzhou, China) was used as the internal control (primers are listed in Table 1).

\section{Identification of miRNA targets}

All target genes and miR-27a binding sites were predicted by MiRanda (http://www.sanger.ac.uk), PicTar (http://pictar.bio.nyu.edu), and TargetScan (http://www.targetscan.org/) and were selected based on a prediction score greater than 0.5 . To identify functional clustering annotations, the lists of candidate target genes were entered into the following web-based tools: Panther (http://www.pantherdb.org), GeneCodis (http://genecodis.dacya.ucm.es/analysis) and Ingenuity (http://www.ingenuity.com).

\section{Luciferase reporter assay}

A dual-luciferase reporter assay was used to confirm that the complementary sequence of miR-27a binds to the 3'-UTR of CDC25B mRNA. The miR-27a binding site (approximately $520 \mathrm{bp}$ ) in the 3'-UTR of CDC25B was amplified by PCR and inserted into the multiple cloning site in PGL3-basic (named "PGL3CDC25B 3'-UTR"). To further verify the binding sites, a fragment containing a complementary nucleotide sequence with miR-27a was inserted into PGL3-basic as a positive control. The HEK293 cells were cultured in 24-well plates and co-transfected with the recombinant reporter plasmid (PGL3-CDC25B 3'-UTR), positive control and miR-27a mimics, separately. The PGL-Renilla vector (Promega) served as a control for transfection with Lipofectamine-2000 (Invitrogen). In addition, $20 \mathrm{nM}$ AllStars siRNA oligos (Qiagen, Suzhou, China) were used was used as a negative control. Luciferase was measured 48 hours after transfection using a dual-luciferase reporter kit according to the manufacturer's instructions (Promega). Firefly luciferase was normalized against Renilla luciferase. All experiments were performed three times with two replicates.

\section{Western blot analyses}

Whole cell lysates of cultured cells were prepared with RIPA buffer (Thermo, Rockford, IL, USA) in the presence of a protease inhibitor or PhosStop cocktail (Roche, Mannheim, Germany). The protein concentration was measured using a BCA protein assay kit (Thermo, Rockford, IL, USA). Western blot analysis was performed as previously described (9), and blots were probed using CDC25B and GAPDH primary antibodies and HRP-conjugated secondary antibodies (MBL, Nagoya, Japan).

\section{KARGER}




\section{Cellular Physiology Cell Physiol Biochem 2018;46:365-374 \begin{tabular}{ll|l} 
DOI: 10.1159/000488436 & Ond Biochemistry & $\begin{array}{l}\text { 2018 The Author(s). Published by S. Karger AG, Basel } \\
\text { www.karger.com/cpb }\end{array}$ \\
\cline { 2 - 3 }
\end{tabular}

Assay of erythroid differentiation: benzidine staining and flow cytometry

The erythroid differentiation of K562 cells were scored by benzidine staining as previously reported [18]. For the detection of intracellular DNA, harvested cells were washed twice with PBS and fixed in 75\% ethanol at $4{ }^{\circ} \mathrm{C}$ overnight. The fixed cells were washed with ice-cold PBS, incubated in RNaseA $(20 \mu \mathrm{g} / \mathrm{mL})$ at $37{ }^{\circ} \mathrm{C}$ for $30 \mathrm{~m}$, and then stained with PI (propidium iodide) $(0.5 \mathrm{mg} / \mathrm{mL})$ at $4{ }^{\circ} \mathrm{C}$ for $30 \mathrm{~min}$. Cells were washed with BSA/PBS (1\%) and resuspended in $500 \mu \mathrm{l}$ PBS. Flow cytometric data detecting PI was acquired from BD FACS Canto II. The average percentages of cells in the G1 and S phase are shown in Fig. 7.

\section{Statistical analysis}

All data are expressed as the mean \pm SD. All results were analyzed using SPSS 17.0 software. Significant differences between the groups were determined by ANOVA, followed by a least significant difference post hoc analysis. A P-value less than 0.05 was considered statistically significant.

\section{Results}

Characterization of miR-27a and $\gamma$-globin expression during erythroid differentiation

K562 cells were widely used as a model for the investigation of erythropoiesis and globin gene expression. K562 cells can be induced by hemin to differentiate into the erythroid lineage. To explore the potential regulatory role of miR-27a in erythropoiesis, K562 cells were induced into erythroid lineage differentiation by hemin treatment. $\gamma$-globin was used as an erythroid-lineage marker to assess the hemin-induced erythroid differentiation. The hemin-induced cells were collected at culture times $0,3,6,12,24$, and 48 hours, and then, the total RNA was extracted to assess the levels of miR-27a and $\gamma$-globin. The mRNA level of $\gamma$-globin gradually increased after 0 hours in the hemin-induced K562 cells and decreased after 24 hours of treatment (Fig. 1A), demonstrating that the K562 cells were committed to the differentiation program. Similarly, the miR-27a levels gradually increased after 0 hours and decreased after the 24hour time point during hemin-induced erythroid differentiation (Fig. 1B). These results indicate that miR-27a may play an important role in erythroid lineage differentiation.

miR-27a regulates hemin-induced erythroid differentiation of $K 562$ cells

Based on the above-mentioned observations, we sought to confirm the biological functions of miR-27a in erythroid differentiation regulation. K562 cells were transfected with Pre-miR27a mimics, and during hemin-induced erythroid differentiation, these cells exhibited markedly higher expression of miR-27a than did the cells transfected with the mimic controls (Fig. 2A). The overexpression of miR-27a clearly upregulated the levels of the erythroidlineage marker $\gamma$-globin in each paired condition (Fig. 2B). In addition, miR-27a was inhibited by the transfection of the miR-27a inhibitor and inhibitor control into the K562 cells. The expression of miR27a was significantly lower in the K562

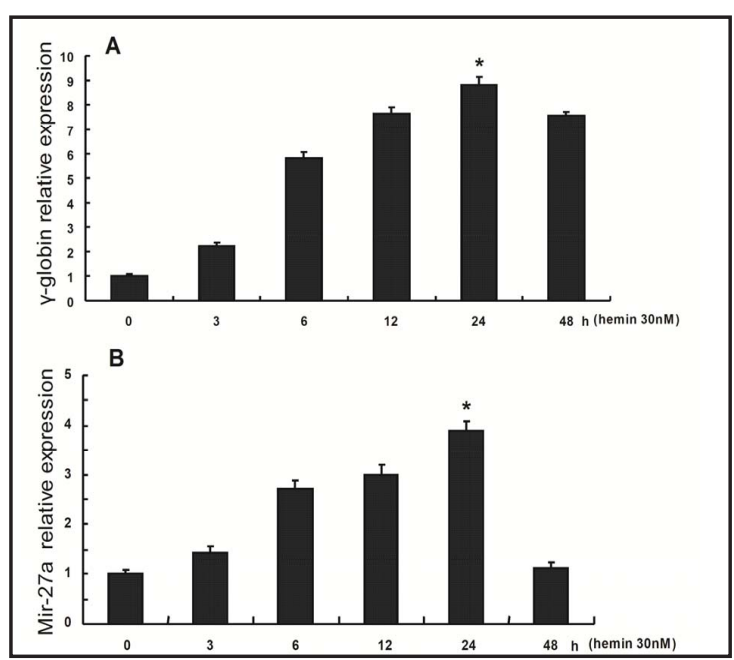

Fig. 1. Expression of miR-27a during erythroid differentiation. A. qRT-PCR analysis of $\gamma$-globin during hemin-induced erythroid differentiation of K562 cells at culture times $0,3,6,12,24$, and 48 hours; B. qRT-PCR analysis of miR-27a during hemin-induced erythroid differentiation of $\mathrm{K} 562$ cells at $0,3,6,12,24$, and 48 hours after induction. GAPDH was used as a control, and the results were normalized to baseline in each experiment. All data represent the mean \pm SD $(n=3)$. ${ }^{*}$ $\leq 0.05$ compared with baseline. 
cells transfected with the miR-27a inhibitor than in the cells transfected with the mimic controls during hemininduced erythroid differentiation (Fig. 2C). In contrast, compared with the inhibitor control treatment, miR27 a inhibition resulted in a lower expression of $\gamma$-globin at each time point after hemin treatment (Fig. 2D). These findings suggested that miR-27a positively regulates the hemin-induced erythroid differentiation of K562 cells.

CDC25B is a target gene of miR-27a in erythropoiesis

CDC25B, a CDC25 phosphatase subtype, is an important regulator of several steps in the cell cycle. Bioinformatics analysis predicted that CDC25B is a direct target of miR$27 \mathrm{a}$, and a complementary miR-27a binding motif is located in the 3'UTR of the CDC25B gene (Fig. 3A). A dual-luciferase reporter assay was performed to determine whether the predicted miR-27a binding motif of CDC25B is a functional target site. The reporter plasmid pGL3-CDC25B 3'-UTR, pre-miR-27a mimics, and positive and negative controls were separately co-transfected into the 293T cells. The luciferase reporter assay demonstrated that compared with the negative transfection control, the co-transfection of pGL3-CDC25B 3'-UTR and the pre-miR-27a mimics resulted in a decrease in firefly luciferase activity, thus demonstrating that miR-27a binds to the 3'-UTR of CDC25B mRNA (Fig. 3B).

\section{CDC25B expression is regulated by} miR-21 in K562 cells.

To validate that CDC25B is a miR27a target, its mRNA and protein levels were analyzed in K562 cells under miR-27a overexpression or suppression. The Pre-miR-27a mimics, mimic control, Pre-miR-27a inhibitor, and inhibitor-NC (scrambled oligonucleotides) were transfected into K562 cells. Consistently, with the results obtained in the dual-luciferase reporter

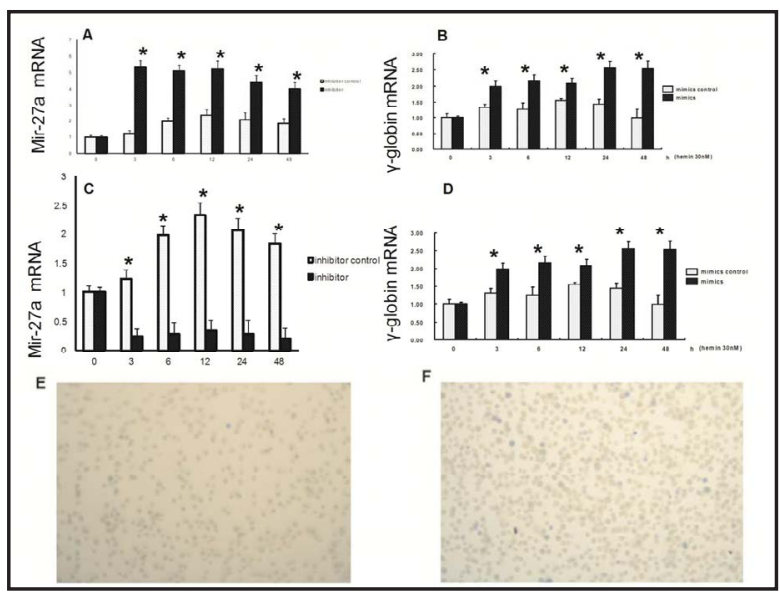

Fig. 2. miR-27a regulates hemin-induced erythroid differentiation. The hemin-induced K562 cells were transiently transfected with Pre-miR-27a mimics, a miR-27a inhibitor or mimic controls and evaluated using real-time qRT-PCR at $0,3,6,12,24$, and 48 hours after induction. All data represent the mean $\pm \mathrm{SD}(\mathrm{n}=3) .{ }^{*} \mathrm{P} \leq 0.05$ compared with the baseline. A. The expression of miR-27a in K562 cells transfected with miR-27a mimics. B. The expression of $\gamma$-globin in K562 cells transiently transfected with miR-27a mimics. C. The expression of miR-27a in K562 cells transfected with miR-27a inhibitor. D. The expression of $\gamma$-globin in K562 cells transfected with miR-27a inhibitor. E and F: Benzidine staining of K562 cells after hemin stimulation. E: mimic control. F: miR-27a mimics.

Fig. 3. $\mathrm{CD}-$ $\mathrm{C} 25 \mathrm{~B}$ is a direct target of miR-27a. A. The highly conserved m i R - 27 a binding motif in the 3'UTR of CDC25B mRNA predicted

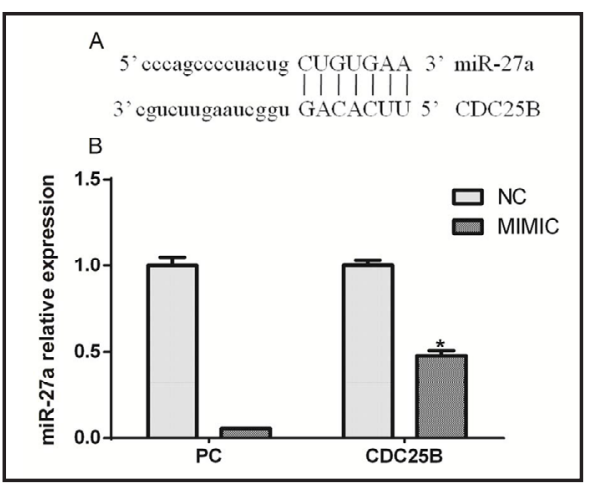
from http://

www.targetscan.org. B. Relative luciferase activity of the indicated CDC25B reporter construct in 293T cells. Cells were co-transfected with pGL3-CDC25B 3'-UTR, pre-miR27a mimics and a PGL-Renilla vector. The positive control (PC, a reporter plasmid containing a complementary nucleotide sequence with miR-27a) and negative control (AllStars siRNA oligos) were also co-transfected. The luciferase assay was performed 48 hours after co-transfection. The data are presented as the mean $\pm S D ; n=3 ;{ }^{*} \mathrm{P}<0.05$ compared with the negative control. 
Fig. 4. CDC25B expression is regulated by miR-27a in K562 cells. A. The effect of pre-miR-27a on the CDC25B mRNA levels. Cells were transfected with Pre-miR-27a mimics, mimic control or Pre-miR27a inhibitor or inhibitor-NC for 48 hours. The relative CDC25B mRNA expression was analyzed by real-time RT-PCR. Expression levels were normalized to those in the negative control siRNA-transfected cells. The data are presented as the mean \pm

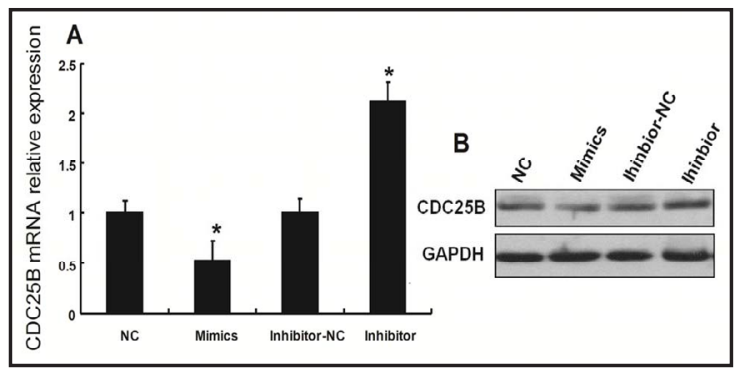
$\mathrm{SD} ; \mathrm{n}=3$; ${ }^{*} \mathrm{P}<0.05$ compared with the negative control. B. The effects of pre-miR-27a on the CDC25B protein level. K562 cells were transfected with Pre-miR27a mimics, Pre-miR-27a inhibitor or inhibitor-NC. The CDC25B protein levels were analyzed by Western blotting 48 hours after the transfection. GAPDH was used as the housekeeping control.

Fig. 5. Expression of CDC25B during erythroid differentiation. Western blotting was used to analyze the changes in CDC25B expression 0, 3, 6, 12, 24, and 48 hours after hemin induction of the K562 cells. GAPDH was used as the housekeeping control.

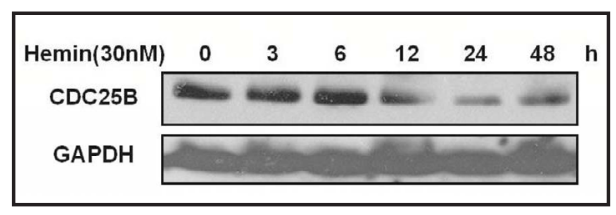

assay, the transient transfection of the Pre-miR-27a mimics significantly decreased the CDC25B transcription levels, and transient transfection of the Pre-miR-27a inhibitor markedly up-regulated the CDC25B mRNA expression in the K562 cells (Fig. 4A). Western blotting further confirmed the abovementioned observations. The CDC25B protein levels were attenuated in miR-27aoverexpressing K562 cells but were increased in the miR-27a-suppressed K562 cells (Fig. 4B).

Decreased CDC25B
expression during
theremin-
induced erythroid
differentiation
To confirm the dynam-

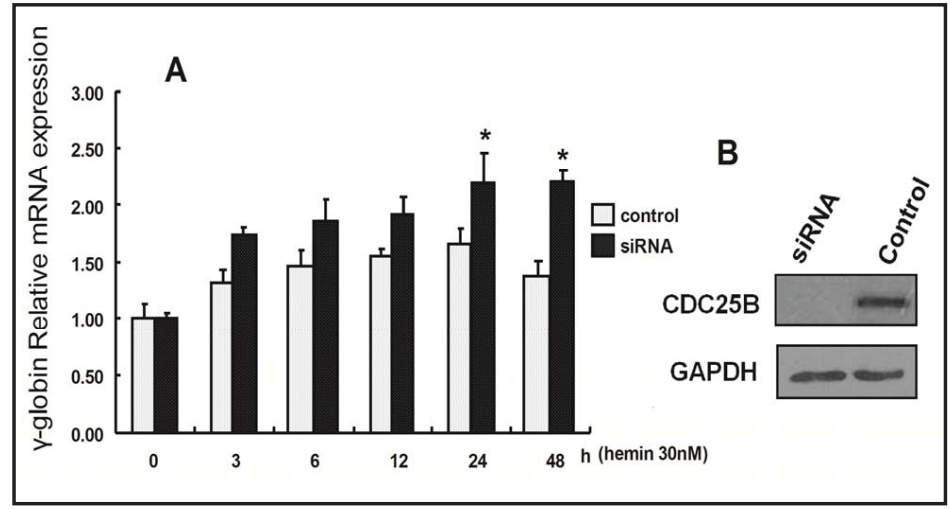

Fig. 6. CDC25B knockout up-regulates $\gamma$-globin expression. A. The effect of CDC25B on $\gamma$-globin mRNA levels. Cells were transfected with CDC25A siRNA and collected at $0,3,6,12,24$, and 48 hours after the hemin induction of the K562 cells. The $\gamma$-globin mRNA levels were analyzed by RT-PCR. The expression levels were normalized to baseline in each experiment. The data are presented as the mean $\pm S D ; n=3$; ${ }^{*} \mathrm{P}<0.05$ compared with the matched siRNA-transfected control cells. B, CDC25B siRNA suppressed the CDC25B expression. The CDC25B protein levels were determined by Western blotting. GAPDH was used as the housekeeping control.

ic variability in CDC25B expression during the hemin-induced erythroid differentiation of K562 cells, we further analyzed the CDC25B protein levels by Western blotting. The hemininduced K562 cells were collected at culture times $0,3,6,12,24$, and 48 hours, and then, the total protein was extracted to assess the CDC25B levels. Western blotting demonstrated that CDC25B gradually decreased after the 0-hour time point in the hemin-induced K562 cells and increased after the 24-hour time point during hemin-induced erythroid differentiation (Fig. 5A, 1B). These results suggest that CDC25B is involved in erythroid lineage differentiation. 


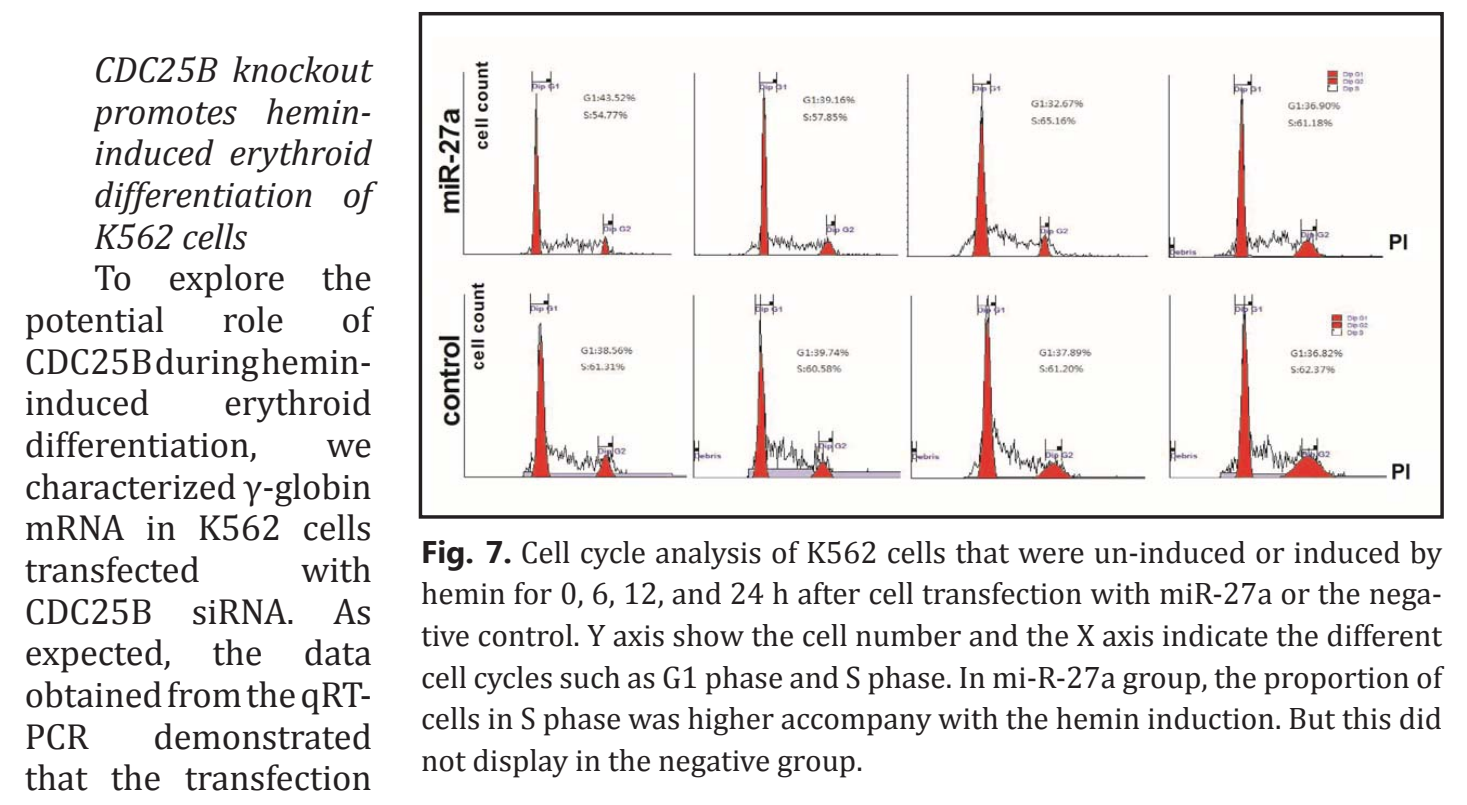

of CDC25B siRNA

significantly suppressed CDC25B expression in the K562 cells (Fig. 6A), and the CDC25B inhibition resulted in increased expression of $\gamma$-globin relative to that in the control group at each time point in the hemin-induced K562 cells (Fig. 6B). Altogether, these findings suggested that CDC25B negatively regulates the hemin-induced erythroid differentiation of K562 cells. Cell cycle analysis also prove that miR-27a could promote K562 cells differentiation (Fig. 7).

\section{MiR-27a overexpression promote cell differentiation}

To elucidate the role of miR-27a during erythroid differentiation, we transfected the miR-27a mimics into the K562 cells, and detected the influence on the cell cycle. In the miR27a transfection group, the proportion of cells in the $S$ phase were higher, along with the hemin induction at different time points. At the same time, there was no change in the control group. This finding indicated that miR-27a could break the cell cycle arrest and induce K562 cell differentiation.

\section{Discussion}

Hematopoiesis is a multistep process leading to the production of mature blood cells and is regulated by numerous factors, including miRNAs. A spatiotemporally distinct set of miRNAs plays an important role in erythroid differentiation [4, 7]. For example, the expression of miR-23a, miR-150 and miR-222 is decreased during erythroid cell development. In addition, miR-210, miR-144, miR-146b and miR-451 are overexpressed during erythroid differentiation $[6,7,10,19]$. MiR-27a is a newly discovered regulatory miRNA that plays an important role in erythroid maturation $[8,10,12]$. Thus, here, we explored the functions and target genes of miR-27a during erythroid differentiation.

In this study, we used K562 cells as an in vitro model of erythropoiesis, because these cells have been proven to be a useful tool for investigating erythroid differentiation and globin gene expression. Hemin is a relatively strong inducer of erythroid differentiation in K562 cells and is used for the treatment of acute porphyria and thalassemia intermedia [20]. To explore the functions of miR-27a in erythroid differentiation, we modulated miR-27a expression in hemin-induced K562 cells and explored the effect of miR-27a on erythroid differentiation.

We first characterized the expression of miR-27a and $\gamma$-globin during erythroid differentiation. $\gamma$-globin increased with the miR-27a overexpression in the hemin-induced K562 cells, thus indicating that the K562 cells were committed to the differentiation program. 


\section{Cellular Physiology Cell Physiol Biochem 2018;46:365-374 \begin{tabular}{l|l} 
and Biochemistry Published online: March 27,2018 & $\begin{array}{l}\text { OO } 2018 \text { The Author(s). Published by s. Karger AG, Basel } \\
\text { www.karger.com/cpb }\end{array}$
\end{tabular}}

Wang et al.: MiR-27a Promotes Erythroid Differentiation by Targeting CDC25B

Our findings are supported by results from several recent studies. For instance, Wang et al. reported that miR-27a is increased in the EPO-induced erythroid differentiation of human CD34+ HPCs [8], and Ma et al. found that miR-27a regulates the $\beta$-like globin gene expression by targeting KLF3 and SP1 [10]. These findings suggest that miR-27a is involved in erythroid differentiation and might play an important role in erythropoiesis.

We proceeded to assess the functions of miR-27a in the regulation of erythroid differentiation by miR-27a gain- and loss-of-function experiments. K562 cells were transfected with Pre-miR-27a mimics, mimic control, miR-27a inhibitor or mimic controls. As expected, the overexpression of miR-27a suppressed hemin-induced erythropoiesis in K562 cells, and specific miR-27a mimics induced the overexpression of the $\gamma$-globin gene. In contrast, hemin-induced erythroid differentiation was significantly suppressed when the K562 cells were transfected with miR-27a inhibitor, which suppressed the expression of the $\gamma$-globin gene. These findings suggested that miR-27a positively regulates the hemininduced erythroid differentiation of K562 cells.

CDC25 phosphatases, including the three isoforms CDC25A, CDC25B and CDC25C, are key regulators in cell cycle progression and are involved in cell fate decisions by activating CDK complexes [14]. CDC25 phosphatases play an important role in erythropoiesis $[17,21]$. Moreover, bioinformatics analysis predicted that CDC25B is a direct target of miR-27a, and the dual-luciferase reporter assay confirmed that CDC25B is a target gene of miR-27a. In many examples, the identification of a specific target of miRNA indicates important cellular events, and altered miRNA expression is a common feature of numerous diseases [22]. In addition, we found that the overexpression of miR-27a decreased the CDC25B mRNA and protein levels in K562 cells. In contrast, miR-27a inhibition up-regulated CDC25B mRNA and protein levels in K562 cells. The major mechanism responsible for the rapid turnover of the CDC25 family of proteins is ubiquitin-mediated proteolysis $[13,15]$. Our findings revealed a novel mechanism of CDC25B mRNA turnover, wherein CDC25B is inactivated by miR-27a in K562 cells. Similarly, this type of miRNA-mediated proteolysis has also been demonstrated in several previous studies, i.e., CDC25A inactivation requires miR-21 and miR-16 [16, 23]. In addition, our study further demonstrated that CDC25B was significantly decreased during erythroid differentiation in hemin-induced K562 cells, and CDC25B siRNA up-regulated the expression of $\gamma$-globin in hemin-induced K562 cells. Altogether, these findings suggested that CDC25B, which is a new target of miR-27a, is involved in the hemin-induced erythroid differentiation of K562 cells.

The activity of the CDC25B family of proteins is tightly regulated by multiple mechanisms during the cell cycle, including phosphorylation, intracellular localization alteration, and interactions with other proteins [15]. Each miRNA may target multiple mRNAs, and multiple miRNAs may target the same mRNA. Given the central role of CDC25B in regulating cell cycle progression, additional mechanisms might potentially fine-tune the activity or level of CDC25B, such as miR-211 [24, 25]. Additionally, the specific association between CDC25B and erythroid differentiation still requires further investigation.

In conclusion, our data demonstrated that miR-27a over expression is coupled with a direct decrease in CDC25B expression. In addition, miR-27a regulates erythroid differentiation via CDC25B in hemin-induced $\mathrm{K} 562$ cells. This report is the first to demonstrate the interaction between miR-27a and CDC25B, thus broadening the understanding of the functions of miRNAs during erythroid differentiation. However, more research is needed to elucidate the upstream regulators, the mechanism underlying the effect of CDC25B, and the correlation between aberrant miR-27a expression and anemic disorders in clinical settings, all of which have rarely been studied.

\section{Acknowledgements}

We thank Fang Wang from Peking Union medical college for the assistance with data analysis. This work was supported by grants from the National Natural Science Foundation 


\section{Cellular Physiology Cell Physiol Biochem 2018;46:365-374

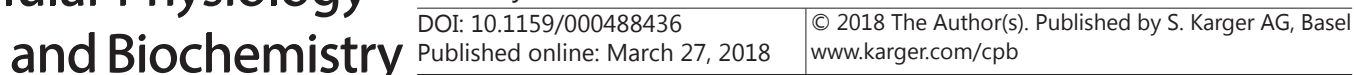

Wang et al.: MiR-27a Promotes Erythroid Differentiation by Targeting CDC25B

of China (no. 81641096), the Department of Science and Technology of Sichuan Province (2016JY0171) and the International S\&T Cooperation Program of China (2015DFA30420).

\section{Disclosure Statement}

We declare no competing financial interests.

\section{References}

Di Leva G, Garofalo M, Croce CM: MicroRNAs in cancer. Annu Rev Pathol 2014;9:287-314.

Ambros V: The functions of animal microRNAs. Nature 2004;431:350-355.

Acunzo M, Romano G, Wernicke D, Croce CM: MicroRNA and cancer--a brief overview. Adv Biol Regul 2015;57:1-9.

4 Azzouzi I, Moest H, Wollscheid B, Schmugge M, Eekels JJM, Speer 0: Deep sequencing and proteomic analysis of the microRNA-induced silencing complex in human red blood cells. Exp Hematol 2015;43:382392.

5 Melkun E, Pilione M, Paulson RF: A naturally occurring point substitution in Cdc25A, and not Fv2/Stk, is associated with altered cell-cycle status of early erythroid progenitor cells. Blood 2002;100:3804-3811.

6 Uehara T, Kondo C, Yamate J, Torii M, Maruyama T: A toxicogenomic approach for identifying biomarkers for myelosuppressive anemia in rats. Toxicology 2011;282:139-145.

7 Sun Z, Wang Y, Han X, Zhao X, Peng Y, Li Y, Peng M, Song J, Wu K, Sun S, Zhou W, Qi B, Zhou C, Chen H, An X, Liu J: miR-150 inhibits terminal erythroid proliferation and differentiation. Oncotarget 2015;6:4303343047.

8 Felli N, Fontana L, Pelosi E, Botta R, Bonci D, Facchiano F, Liuzzi F, Lulli V, Morsilli O, Santoro S, Valtieri M, Calin GA, Liu CG, Sorrentino A, Croce CM, Peschle C: MicroRNAs 221 and 222 inhibit normal erythropoiesis and erythroleukemic cell growth via kit receptor down-modulation. Proc Natl Acad Sci U S A 2005;102:18081-18086.

-9 Yu XY, Zhang Z, Liu J, Zhan B, Kong CZ: MicroRNA-141 is downregulated in human renal cell carcinoma and regulates cell survival by targeting CDC25B. Onco Targets Ther 2013;6:349-354.

10 Zhang YC, Ye H, Zeng Z, Chin YE, Huang YN, Fu GH: The NF-kappaB p65/miR-23a-27a-24 cluster is a target for leukemia treatment. Oncotarget 2015;6:33554-33567.

-11 Song GQ, Zhao Y: MicroRNA-211, a direct negative regulator of CDC25B expression, inhibits triple-negative breast cancer cells' growth and migration. Tumour Biol 2015;36:5001-5009.

12 Wang P, Zou F, Zhang X, Li H, Dulak A, Tomko RJ, Jr., Lazo JS, Wang Z, Zhang L, Yu J: microRNA-21 negatively regulates Cdc25A and cell cycle progression in colon cancer cells. Cancer Res 2009;69:8157-8165.

13 Wang F, Zhu Y, Guo L, Dong L, Liu H, Yin H, Zhang Z, Li Y, Liu C, Ma Y, Song W, He A, Wang Q Wang L, Zhang J, Li J, Yu J: A regulatory circuit comprising GATA1/2 switch and microRNA-27a/24 promotes erythropoiesis. Nucleic Acids Res 2014;42:442-457.

14 Zhang L, Sankaran VG, Lodish HF: MicroRNAs in erythroid and megakaryocytic differentiation and megakaryocyte-erythroid progenitor lineage commitment. Leukemia 2012;26:2310-2316.

15 Bianchi N, Zuccato C, Finotti A, Lampronti I, Borgatti M, Gambari R: Involvement of miRNA in erythroid differentiation. Epigenomics 2012;4:51-65.

16 Shiraishi M, Yamamoto Y, Hirooka N, Obuchi Y, Tachibana S, Makishima M, Tanaka Y: A high concentration of triiodothyronine attenuates the stimulatory effect on hemin-induced erythroid differentiation of human erythroleukemia K562 cells. Endocr J 2015;62:431-440.

17 Cooper MC, Levy J, Cantor LN, Marks PA, Rifkind RA: The effect of erythropoietin on colonial growth of erythroid precursor cells in vitro. Proc Natl Acad Sci U S A 1974;71:1677-1680.

18 Zhu Y, Wang D, Wang F, Li T, Dong L, Liu H, Ma Y, Jiang F, Yin H, Yan W, Luo M, Tang Z, Zhang G, Wang Q Zhang J, Zhou J, Yu J: A comprehensive analysis of GATA-1-regulated miRNAs reveals miR-23a to be a positive modulator of erythropoiesis. Nucleic Acids Res 2013;41:4129-4143. 


\section{Cellular Physiology Cell Physiol Biochem 2018:46:365-374

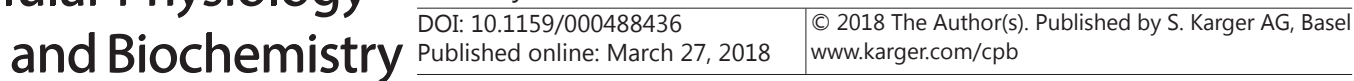

Wang et al.: MiR-27a Promotes Erythroid Differentiation by Targeting CDC25B

19 Ma Y, Wang B, Jiang F, Wang D, Liu H, Yan Y, Dong H, Wang F, Gong B, Zhu Y, Dong L, Yin H, Zhang Z, Zhao H, Wu Z, Zhang J, Zhou J, Yu J: A feedback loop consisting of microRNA 23a/27a and the beta-like globin suppressors KLF3 and SP1 regulates globin gene expression. Mol Cell Biol 2013;33:3994-4007.

20 Agius E, Bel-Vialar S, Bonnet F, Pituello F: Cell cycle and cell fate in the developing nervous system: the role of CDC25B phosphatase. Cell Tissue Res 2015;359:201-213.

-21 Su R, Dong L, Zou D, Zhao H, Ren Y, Li F, Yi P, Li L, Zhu Y, Ma Y, Wang J, Wang F, Yu J: microRNA-23a, -27a and -24 synergistically regulate JAK1/Stat3 cascade and serve as novel therapeutic targets in human acute erythroid leukemia. Oncogene 2016;35:6001-6014.

-22 Xie L, Jing R, Qi J, Lin Z, Ju S: Drug resistance-related microRNAs in hematological malignancies: translating basic evidence into therapeutic strategies. Blood Rev 2015;29:33-44.

23 Brenner AK, Reikvam H, Lavecchia A, Bruserud O: Therapeutic targeting the cell division cycle 25 (CDC25) phosphatases in human acute myeloid leukemia--the possibility to target several kinases through inhibition of the various CDC25 isoforms. Molecules 2014;19:18414-18447.

24 Boutros R, Lobjois V, Ducommun B: CDC25 phosphatases in cancer cells: key players? Good targets? Nat Rev Cancer 2007;7:495-507.

25 Bavelloni A, Poli A, Fiume R, Blalock W, Matteucci A, Ramazzotti G, McCubrey JA, Cocco L, Faenza I: PLCbeta 1 regulates the expression of miR-210 during mithramycin-mediated erythroid differentiation in K562 cells. Oncotarget 2014;5:4222-4231. 